American Journal of Applied Sciences 9 (4): 605-608, 2012

ISSN 1546-9239

(C) 2012 Science Publications

\title{
Low Pass Filter Model for Chemical Sensors in Response to Gases and Odors
}

\author{
Mahmoud Z. Iskandarani \\ Department of Computer Science, \\ Faculty of Science and Information Technology, \\ Al-Zaytoonah University of Jordan
}

\begin{abstract}
Design and Modeling multi-gap sensing odor system for the objectives of odor recognition, classification and correlation are carried out. The model illustrates the low pass functionality of the multi-gap sensor acting as a filter for odors. Problem statement: Odor filtering is an important issue in today's world. In addition knowing the original material that an odor belongs to even after being mixed with others is also of vital importance. In addition measuring quality of mixed odors in terms of their affinity and belonging to a specific category or is critical. Approach: Mathematical modeling using low pass filter is carried out. Results: Clear evidence of ability to filter components of an odor mixture as the multi-gap sensor is acting as a filter. Conclusion: The ability to custom design chemical sensors to indicate the presence of various odors.
\end{abstract}

Key words: Odor sensor, odor discrimination, multi-gap, gas sensing, pass functionality, multi-gap chemical, two-dimensional freedom, limited test duration, enthalpy resulting

\section{INTRODUCTION}

Biological sensing systems have an excellent capabilities extracting very specific information from an array of inputs. It is assumed that such sensory processing capability would consist of highly precise sensors as the fundamental building blocks of the processing systems. However, it is not the case. Sensing and discrimination in biological systems rely on large arrays to provide redundancy, overcome low precision in individual sensors with good interconnectivity to provide comprehensive data coupled with diverse topology.

Some standalone chemical sensors suffer many problems with reproducibility, selectivity, sensitivity, stability and response time.

The individual chemical sensor is successful in detecting particular chemicals. However, in sensing environments that are potentially visited by chemical mixtures, false readings can often occur. Such discrimination errors, leading to poor selectivity are associated with many chemical sensors. Most sensors are inherently sensitive to a wide variety of chemicals. Part of the solution is the implementation of arrays of chemical sensors; designed properly with appropriate signal processing techniques.

Since the first applications of solid state gas sensors in arrays, electronic noses went through a great deal of development. Many articles on this subject have been published over the last few years, mainly in relation to the food and beverage industry, but also concerning environmental, agricultural, medical topics and automotive industry, among others. However, the number of studies dedicated to nose based odor originality systems is still very limited, probably due to the complexity of their matrices (Wongchoosuk et al., 2009; Myrick et al., 2009; Tang et al., 2010; Jha and Yaava, 2011; Bucak and Karlik, 2009; Huang and Wan, 2009; Zeng et al., 2009; Lange et al., 2009; Vera et al., 2011; Song et al., 2011; Cole et al., 2011; Im et al., 2011; Baldwin et al., 2011; Huan et al., 2011; Phaisangittisagul and Nagle, 2011).

In this study modeling of the functionality of multigap chemical sensors is carried out using a low pass filtering model.

\section{MATERIALS AND METHODS}

Monitoring the composition of atmospheric gases is very important requirement for personal protection and environmental monitoring. The results of dangerous gas concentrations are usually needed immediately, with a low false-alarm tolerance, high sensitivity and minimum cost. Multi-Gap chemical sensors with a specific gap-ratio sensor structure, although clearly not perfect, offer the potential to achieve those goals.

Multi-Gap chemical sensors are very attractive because of their remarkable sensitivity due to changes 
of the boundary conditions caused by the interaction of a layered structure with specific gas molecules.

The extent to which gas is adsorbed by a solid depends on the following factors:

- The physical and chemical nature of the gas

- The physical and chemical nature of the solid (the important feature being the specific surface area and the pore size distribution)

- Temperature

- Pressure

The adsorption of gases or vapors on solids involves:

Physical adsorption: The adsorbed gas is held to the solid surface by weak, non-specific Van der Waals forces. Any gas will physically adsorb on any solid surface to some extent. Adsorption equilibrium is attained rapidly and the process is easily reversible. The activation energy of physical adsorption is Zero. Physical adsorption is, therefore, most prominent at low temperatures. Also, the enthalpy of adsorption is low; hence the adsorbed gas may have some twodimensional freedom with the possibility of multi-layer adsorption.

Chemisorption: The adsorbed gas is held to the solid surface by strong, specific chemical bonding. Adsorption equilibrium is established slowly, owing to the high activation energy that is usually involved. Chemisorption is, therefore, most prominent at high temperatures with high enthalpy. The adsorption will tend to be localized and only monolayer adsorption is possible. Gas adsorption involves loss of translational freedom with a negative change in free energy and enthalpy resulting in an exothermic process. Increasing temperature, results in an increase in the kinetic energy of the gas, which favours desorption. This means that the equilibrium amount of adsorbed gason a solid decreases with temperature (Haddi et al., 2011; Barbri et al., 2008; Alizadeh, 2010; Musatov et al., 2010).

The model is based on the observation that the transient response of the tested multi-gap sensors has the same slope as the impulse response of a low-pass filter at a specific gas concentration and has an equivalent overall output characteristic as the step response of a low-pass filter. This observation is supported by the fact that the $\mathrm{PbPc}$ material is deposited over gold electrodes with ohmic contacts and the assumption that the multi-gap design may result in the sublimed film material acting as a dielectric between two golden electrodes. Also, from experimental data, change in the conductivity of the designed sensors is related to time response through Eq. 1 and 2:

$$
\mathrm{G}=\mathrm{G}_{\text {initial }}+\mathrm{G}_{\text {SteadsState }}\left(1-\exp \left(-\frac{\mathrm{t}^{*}}{\tau}\right)\right)
$$

Then:

$$
\left(\mathrm{G}-\mathrm{G}_{\text {initial }}\right)=\mathrm{G}_{\text {Steadystate }}\left(1-\exp \left(-\frac{\mathrm{t}^{*}}{\tau}\right)\right)
$$

Where:

$\mathrm{t}^{*} \quad=$ Elapsed time for each applied gas concentration to reach saturation point rearranged in reverse order to compensate for the limited test duration and to truly represent impulse and step responses.

$\tau \quad=$ Time Constant

$\mathrm{G}_{\text {Steadystak }}=$ Conductance final value

Hence, both impulse and step response need to be incorporated in a way that provides a simple model representing sensor's response.

From Eq. 2, we can consider the effect of the following factors on device conductance.

\section{Gap width:}

$\left(\mathrm{G}-\mathrm{G}_{\text {initial }}\right)=\left[\left(\mathrm{G}_{\text {Steadystate }} \cdot \mathrm{f}(\mathrm{W})\right)\left(1-\exp \left(-\frac{\mathrm{t} *}{\tau}\right)\right)\right]$

where, $\mathrm{f}(\mathrm{W})$ a factor obtained from the provided tables to account for gap width and is given by:

$f(W)=\left.\left(\frac{W(n+1)}{W(n)}\right)\right|_{n=1,2, \ldots M}$

where, n: gap number.

\section{Temperature:}

$\left(\mathrm{G}-\mathrm{G}_{\text {initial }}\right)=\left[\left(\mathrm{G}_{\text {SteadyState }} \cdot \mathrm{f}(\mathrm{T})\right)\left(1-\exp \left(-\frac{\mathrm{t} *}{\tau}\right)\right)\right]$

where, $\mathrm{f}(\mathrm{T})$ a factor obtained from the provided tables to account for temperature change and is given by:

$f(T)=\left.\left(\frac{T(k+1)}{T(k)}\right)\right|_{k=1,2, \ldots M}$ 
where, $\mathrm{k}$ : temperature number.

Film thickness:

$$
\left(\mathrm{G}-\mathrm{G}_{\text {initial }}\right)=\left[\left(\mathrm{G}_{\text {SteadyState }} \cdot \mathrm{f}(\mathrm{y})\right)\left(1-\exp \left(-\frac{\mathrm{t} *}{\tau}\right)\right)\right]
$$

where, $f(y)$ a factor obtained from the provided tables to account for film thickness change and is given by:

$f(y)=\left.\left(\frac{y(L+1)}{y(L)}\right)\right|_{L=1,2, \ldots M}$

where, $\mathrm{L}$ is film thickness number.

\section{RESULTS}

Considering the previous equations, the reference numbers are the smallest values. Hence:

Gap width: reference gap width is gap1:

$\mathrm{n}=1 \Rightarrow \mathrm{n}+1=2 \Rightarrow$ Gap

$2 \mathrm{n}=2 \Rightarrow \mathrm{n}+2=2 \Rightarrow$ Gap3

Temperature: Reference temperature is temp1 = $130^{\circ} \mathrm{C}$ :

$\mathrm{k}=1 \Rightarrow \mathrm{k}+1=2 \Rightarrow \mathrm{T}_{2}=160$

$\mathrm{k}=2 \Rightarrow \mathrm{k}+1=3 \Rightarrow \mathrm{T}_{3}=190$

Film thickness: Reference thickness is $\mathrm{y}_{1}=500 \mathrm{~nm}$ :

$$
\begin{aligned}
& y=1 \Rightarrow y+1=2 \Rightarrow y_{2}=1000 \mathrm{~nm} \\
& y=2 \Rightarrow y+1=3 \Rightarrow y_{3}=1500 \mathrm{~nm}
\end{aligned}
$$

All the previous is tied together in the model. To eliminate the different variable and obtain a final expression relation sensor conductance to time, conductance ration, which results in Eq. 3:

$$
\Delta \mathrm{G}_{\text {Ratio }}=\mathrm{G}_{\text {Ratio }}\left(1-\exp \left(-\frac{\mathrm{t}^{*}}{\tau}\right)\right)
$$

\section{DISCUSSION}

Figure 1 show an obtained three gap system, with Fig. 2 showing a comparative response curves which agrees with the presented expressions. The gap ratio used is $1: 3.3: 10$. From the figures, it is obvious the effect of inter-electrode separation has on the overall conductivity, hence a change in the overall conductance and sensor response to applied gases.

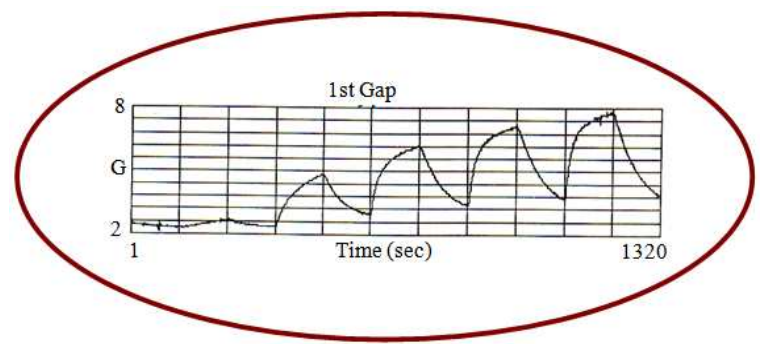

(a)

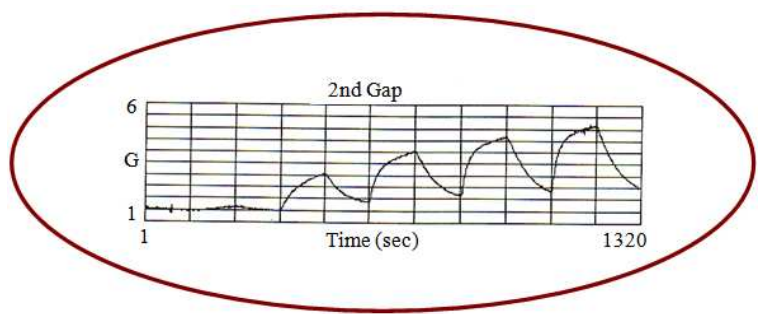

(b)

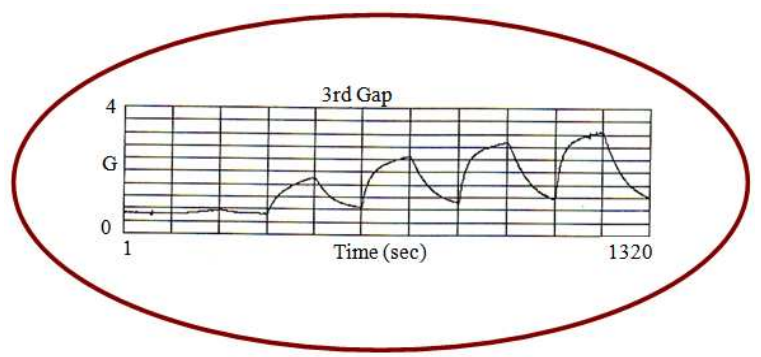

(c)

Fig. 1: Gap size effect on chemical sensor response

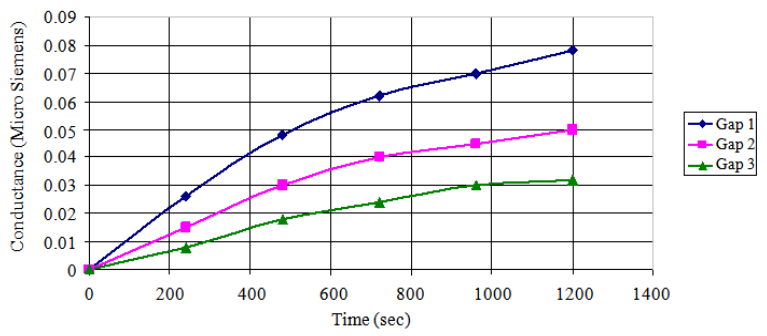

Fig. 2: Comparative response of three-gap sensory system 


\section{CONCLUSION}

A low pass filter model show the possibility to custom design and build chemical sensors based on previous calculations. This allows research into odors and chemical to become more pointed and objective oriented.

\section{REFERENCES}

Alizadeh, T., 2010. Chemiresistor sensors array optimization by using the method of coupled statistical techniques and its application as an electronic nose for some organic vapors recognition. Sensors Actuators B: Chem., 143: 740-749. DOI: 10.1016/j.snb.2009.10.018

Baldwin, E.A., J. Bai, A. Plotto and S. Dea, 2011. Electronic noses and tongues: Applications for the food and pharmaceutical industries. Sensors, 11: 4744-4766. DOI: 10.3390/s110504744

Barbri, N.E., E. Llobet, N.E. Bari, X. Correig and B. Bouchikhi, 2008. Electronic nose based on metal oxide semiconductor sensors as an alternative technique for the spoilage classification of red meat. Sensors, 8: 142-156: DOI: 10.3390/s8010142

Bucak, I.O. and B. Karlik, 2009. Hazardous odor recognition by CMAC Based neural networks. Sensors, 9: 7308-7319. DOI: 10.3390/s90907308

Cole, M., J.A. Covington and J.W. Gardner, 2011. Combined electronic nose and tongue for a flavour sensing system. Sensors Actuators B: Chem., 156: 832-839. DOI: $10.1016 / j . s n b .2011 .02 .049$

Haddi, Z., A. Amari, H. Alami, N.E. Bari and E. Llobet et al., 2011. A portable electronic nose system for the identification of cannabis-based drugs. Sensors Actuators B: Chem., 155: 456-463. DOI: 10.1016/j.snb.2010.12.047

Huan, C., L. Zhiyu and F. Gang, 2011. Analysis of the aging characteristics of $\mathrm{SnO}_{2}$ gas sensors. Sensors Actuators B: Chem., 156: 912-917. DOI: 10.1016/j.snb.2011.03.005

Huang, J. and Q. Wan, 2009. Gas sensors based on semiconducting metal oxide one-dimensional nanostructures. Sensors, 9: 9903-9924. DOI: 10.3390/s91209903

Im, J., S.K. Sengupta, M.F. Baruch, C.D. Granz and S. Ammu et al., 2011. A hybrid chemiresistive sensor system for the detection of organic vapors. Sensors Actuators B: Chem., 156: 715-722. DOI: 10.1016/j.snb.2011.02.025
Jha, S.K. and R.D.S. Yaava, 2011. Power scaling of chemiresistive sensor array data for odor classification. J. Patt. Recog. Res., 6: 65-74. www.jprr.org/index.php/jprr/article/view/247

Lange, U., N.V. Roznyatovskaya and V.M Mirsky, 2009. Conducting polymers in chemical sensors and arrays. Anal. Chim. Acta, 614: 1-26. DOI: 10.1016/j.aca.2008.02.068

Musatov, V.Y., V.V. Sysoev, M. Sommer and I. Kiselev, 2010. Assessment of meat freshness with metal oxide sensor microarray electronic nose: A practical approach. Sensors Actuators B: Chem., 144: 99-103. DOI: 10.1016/j.snb.2009.10.040

Myrick, A.J., K.C. Park, J.R. Hetling and T.C. Baker, 2009. Detection and discrimination of mixed odor strands in overlapping plumes using an insectantenna-based chemosensor system. J. Chem. Ecol., 35: 118-130. DOI: 10.1007/s10886-0089582-4

Phaisangittisagul, E. and H.T. Nagle, 2011. Predicting odor mixture's responses on machine olfaction sensors. Sensors Actuators B: Chem., 155: 473482. DOI: $10.1016 /$ j.snb.2010.12.049

Song, K., Q. Liu and Q. Wang, 2011. Olfaction and hearing based mobile robot navigation for odor/sound source search. Sensors, 11: 2129-2154. DOI: $10.3390 / \mathrm{s} 110202129$

Tang, K.T., S.W. Chiu, C.H. Pan, H.Y. Hsieh and Y.S. Liang et al., 2010. Development of a portable electronic nose system for the detection and classification of fruity odors. Sensors, 10: 91799193. DOI: $10.3390 / \mathrm{s} 101009179$

Vera, L., L. Acena, J. Guasch, R. Boque and M. Mestres et al., 2011. Characterization and classification of the aroma of beer samples by means of an MS e-nose and chemometric tools. Anal. Bioanalytical Chem., 399: 2073-2081. DOI: 10.1007/s00216-010-4343-y

Wongchoosuk, C. M. Lutz and T. Kerdcharoen, 2009. Detection and classification of human body odor using an electronic nose. Sensors, 9: 7234-7249. DOI: 10.3390/s90907234

Zeng, W., T. Liu, Z. Wang, S. Tsukimoto and M. Saito et al., 2009. Selective detection of formaldehyde gas using a Cd-Doped $\mathrm{TiO}_{2}-\mathrm{SnO}_{2}$ Sensor. Sensors, 9: 9029-9038. DOI: 10.3390/s91109029 